\title{
A screw microdrive for adjustable chronic unit recording in monkeys
}

\author{
Altah M. Nichols, Thomas W. Ruffner, Marc A. Sommer*, Robert H. Wurtz \\ Laboratory of Sensorimotor Research, Building 49, Room 2A50, National Eye Institute, National Institutes of Health, Bethesda, \\ MD 20892-4435, USA
}

Received 24 October 1997; received in revised form 3 February 1998; accepted 3 February 1998

\begin{abstract}
A screw microdrive is described that attaches to the grid system used for recording single neurons from brains of awake behaving monkeys. Multiple screwdrives can be mounted on a grid over a single cranial opening. This method allows many electrodes to be implanted chronically in the brain and adjusted as needed to maintain isolation. ( $) 1998$ Elsevier Science B.V. All rights reserved.
\end{abstract}

Keywords: Monkey; Single unit recording; Chronic recording; Grid system; Microdrive; Screwdrive

\section{Introduction}

A grid system for neuronal recording from awake behaving monkeys (Crist et al., 1988) facilitates the systematic exploration of a region of the brain. The grid fits into a cylinder that is permanently implanted over a cranial opening. Two methods of recording have been used with the grid system. First, microelectrodes have been advanced through the dura, into the brain, on a daily basis using a hydraulic or stepper microdrive temporarily attached to the cylinder. This allows the electrode depth to be precisely adjusted, but the electrode must be removed at the end of the day. Alternatively, electrodes have been positioned in the brain and then fixed to the grid. This allows the electrode to remain in place indefinitely, but it cannot be repositioned.

We have recently designed and used a small, simple screw microdrive for the grid system that allows chronically implanted electrodes to be repositioned as needed. The screwdrive attaches to the grid, within the cylinder, and holds one or more electrodes that access

\footnotetext{
* Corresponding author. Tel.: + 1301 4961141; fax: + 1301 4020511; e-mail: mas@1sr.nei.nih.gov
}

the brain through a guidetube. Electrodes are advanced or retracted by turning the screw. Several screwdrives may be mounted on the same grid. This device is derived from the drives used for chronic recording in rats by McNaughton and colleagues (Wilson and McNaughton, 1993; Gothard et al., 1996).

\section{Materials and methods}

Figs. 1 and 2 show schematics and oblique views of two versions of the screwdrive. All components are stainless steel. The screwdrives are made from threaded rod $(1 \mathrm{~mm} \times 0.25 \mathrm{pitch})$ that is commercially available (HiTech Fasteners, Frederick, MD). The lower end of the rod, with threads removed, sits in a hole and can rotate. A small block that holds the electrode is threaded onto the upper portion of the rod. When one turns the rod with a jeweler's screwdriver, the block and electrode move vertically, perpendicularly to the grid. Rotation of the block and electrode is minimized with supporting rods. The electrode is attached to the moving block with water-resistant epoxy (Devcon Plastic Welder, Read Plastics, Rockville, MD) or dental acrylic (Lang Dental, Wheeling, IL). 


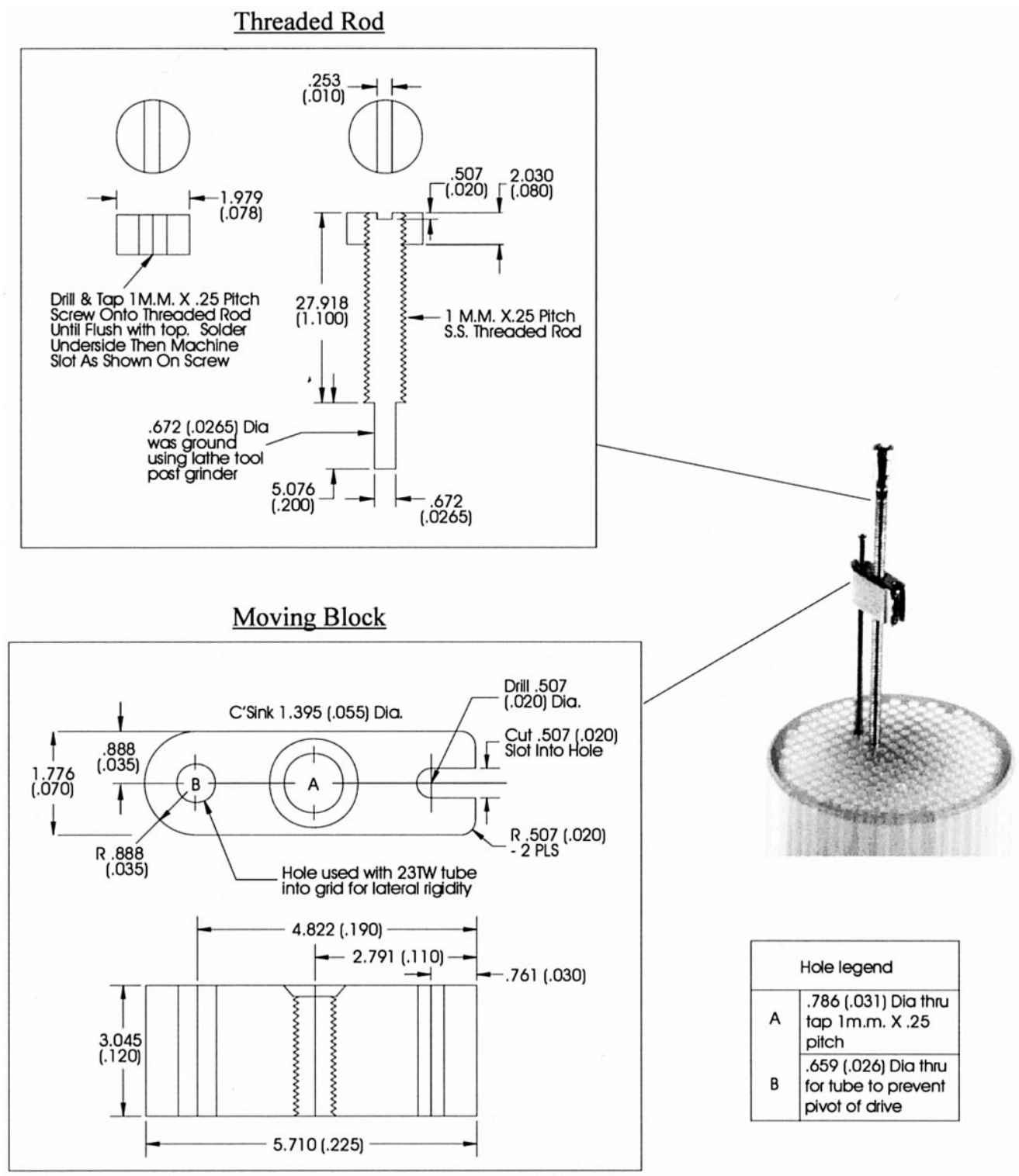

Fig. 1. The screwdrive that is held by a force fit into a grid hole. In the schematics, dimensions are given both in mm and, parenthesized, in inches. In the oblique view, scale is provided by the grid hole separations of $1 \mathrm{~mm}$ in cardinal directions. For clarity, the guide tube and electrode are not shown. The guide tube would be inserted in a grid hole directly under the moving block's notch (see oblique view), and the electrode would be inserted into the guide tube and cemented to the notch.

Each full turn of the screw advances the electrode by 250 microns. Since we were able to rotate the screw accurately by as little as $1 / 8$ turn, we could easily advance the microelectrode in steps as small as $\sim 30$ microns.

In one version of the screwdrive (Fig. 1), the rod sits and rotates in a grid hole. This screwdrive has the advantage of ease of fabrication but the disadvantage of possible instability over time because of the friction fit into the plastic grid hole. In the second version (Fig. 2), the threaded rod sits and rotates in the hole of a small base plate that is attached firmly to the grid by a stainless steel screw (\# $000 \times 5 / 32$ self tapping, Morris Precision Screws and Parts, Southbridge, MA). A top piece and two support rods provide full stabilization of the screwdrive. This version has the advantage of an immovable attachment to the grid but the disadvantage of requiring increased fabrication time and expense.

\section{Discussion}

We have used the screwdrives (unpublished experiments) to record from monkey superior colliculus and frontal cortex with standard tungsten microelectrodes (FHC, Bowdoinam, ME) or bundles of eight microwires (NB LABS, Denison, TX). The ability to adjust the depth of chronically placed electrodes al- 


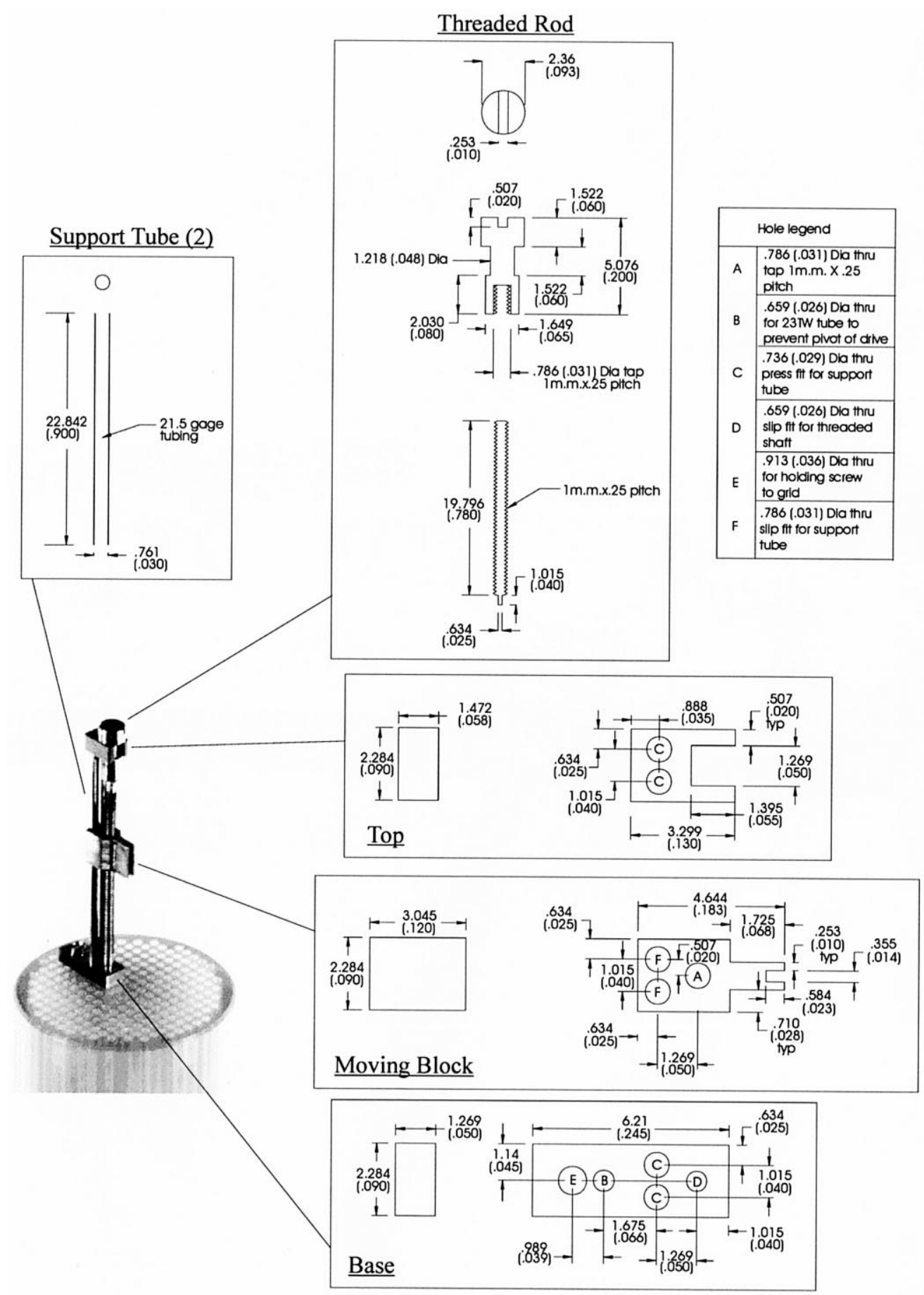

Fig. 2. The screwdrive that is attached to the grid by a base plate. See Fig. 1 legend for details.

lowed us to continue isolating new units on the implanted electrodes over periods of weeks. This was a substantial improvement over fixing electrodes in place, which put our experiments at the mercy of a single recording location and the gradual decrease in the signal-to-noise ratio that usually occurred there.

We came to prefer the drive that was firmly attached to the grid with a base piece (Fig. 2). The two support rods provided superior rigidity as the screw was being turned, such that rotation of the moving block was negligible. Furthermore, if the moving block was lowered so much that it hit the top of the electrode's guide tube, one became immediately aware of this: the vertical immobility of this screwdrive made it impossible to turn the screw any more. When the first version of the screwdrive (Fig. 1) was used, collision between the moving block and guide tube was hard to detect. The screw could still be turned, but because the block could 
not descend further, the entire screwdrive would start to rise out of the grid hole. Despite the drawbacks of this first version (Fig. 1), we include it in this note because we found it to be useful in pilot studies and other experiments that necessitated quick fabrication of multiple screwdrives.

Several procedures were used to maintain the preparation when screwdrives were attached to the grid for weeks. First, we drilled two drain holes (approx. $3 \mathrm{~mm}$ radius) in the grid to allow us to irrigate between the underside of the grid and the dura using saline and hydrogen peroxide. Second, we placed a dab of antibiotic ointment on the top of the electrode's guidetube at the end of every recording day. Third, we attempted to keep the threads over which the moving block traveled as clean as possible, because obstructed threads made the advance irregular. Finally, because the screwdrives stand higher than the walls of our recording cylinders and prevent attachment of a normal cylinder cap, we sealed the preparation at the end of every session by using a taller cylinder cap or by fastening an extension cylinder between the recording cylinder and a normal cap.

We have not found the screwdrive to be an attractive alternative to a conventional hydraulic or stepper microdrive when continuous adjustment of depth is required, such as when searching for a particular brain area; the time and effort required to turn the screw is prohibitive. Where we have found the screwdrive to be exceptionally useful is for the slight adjustments re- quired for electrodes that are present on a long-term basis and/or when a number of drives are required within the same grid.

\section{Note added in proof}

It recently came to our attention that a similar design has been developed by Earl Miller at MIT. He uses a moving block that is crafted from dental acrylic, which reduces expense and machine shop time.

\section{Acknowledgements}

We are grateful for the continuing suggestions of Cynthia Erickson throughout our experiments using these screwdrives. We thank Richard Krauzlis for helping to test the second version (Fig. 2).

\section{References}

Crist CF, Yamasaki DSG, Komatsu H, Wurtz RH. A grid system and a microsyringe for single cell recording. J Neurosci Methods 1988;26:117-22.

Gothard KM, Skaggs WE, Moore KM, McNaughton BL. Binding of hippocampal CA1 neural activity to multiple reference frames in a landmark-based navigation task. J Neurosci 1996;16:823-35.

Wilson MA, McNaughton BL. Dynamics of the hippocampal ensemble code for space. Science 1993;261:1055-8. 